\title{
Dosimetric Comparison of Intensity-Modulated Radiotherapy versus Three-Dimensional Conformal Radiotherapy for Patients with Brain Tumors
}

\author{
Doaa M. Al Zayat ${ }^{*}$, Ehab M. Attalla ${ }^{2 *}$, Hassan S. Abouelenein ${ }^{3}$, Yasser M. Elkem4, \\ Wafaa Khalil ${ }^{5}$ \\ ${ }^{1}$ Ayadi Al mostakbl Oncology Center, Alex, Egypt \\ ${ }^{2}$ National Cancer Institute, Cairo University, Cairo, Egypt \\ ${ }^{3}$ Children's Cancer Hospital, Cairo, Egypt \\ ${ }^{4}$ Cancer Management and Research Department, Medical Research Institute, Alex University, Alex, Egypt \\ ${ }^{5}$ Faculty of Science, Cairo University, Cairo, Egypt \\ Email: doaazayat@yahoo.com, ${ }^{*}$ attalla.ehab@gmail.com
}

Received 1 January 2014; revised 1 February 2014; accepted 8 February 2014

Copyright $@ 2014$ by authors and Scientific Research Publishing Inc.

This work is licensed under the Creative Commons Attribution International License (CC BY).

http://creativecommons.org/licenses/by/4.0/

(c) (1) Open Access

\section{Abstract}

Purpose: To compare target dose distribution and dose to normal tissue for brain tumors using intensity-modulated and three-dimensional conformal radiotherapy. Methods and Materials: Twelve patients selected in the present study compared the dosimetry of intensity-modulated radiation therapy (IMRT) and three-dimensional conformal radiation therapy (3DCRT) techniques in patients treated with different diagnosis brain tumor. All patients underwent computed tomography planning in conjunction with magnetic resonance imaging fusion. Prescription dose and normal-tissue constraints were identical for both plans. The tolerance level for maximum dose was 6.0 Gy for lenses and 54.0 Gy for brain stem, and optical nerves. Target coverage was evaluated with the D98\%, D95\% and D2\%. Normal tissue dose was evaluated with the maximum dose to lenses, brain stem, and optical nerves. Results: Mean and standard deviation values of PTV 98\%, PTV 95\% and PTV 2\% in IMRT these values were $94.6 \pm 2.3,96.56 \pm 1.67$ and $106.5 \pm 1.87$ respectively; for 3DCRT were $92.7 \pm 3.09,95.58 \pm 1.24$ and $104.45 \pm 2.73$ respectively. IMRT plan provided reduced dose to brainstem $(72.17 \pm 29.967)$ and lenses $(2.43 \pm 2.39)$ relative to the 3DCRT plans for brainstem (73.05 \pm 34.59$)$ and lenses $(7.65 \pm 23.24)$. But optic nerves with IMRT

\footnotetext{
"Corresponding authors.
}

How to cite this paper: Al Zayat, D.M., et al. (2014) Dosimetric Comparison of Intensity-Modulated Radiotherapy versus Three-Dimensional Conformal Radiotherapy for Patients with Brain Tumors. Open Journal of Radiology, 4, 85-96. 
significantly received higher doses $(38.86 \pm 32.15)$ than 3D-CRT $(27.57 \pm 31.45)$. Conclusion: Dose coverage of the planning target volume (PTV) and organ at risk (OAR) was better with IMRT. If PTV is distant to optical nerves and brainstem 3DCRT technique can be applied, and if the PTV is nearby OAR intensity-modulated treatment technique should be used.

\section{Keywords}

\section{Brain Cancer; 3DCRT; IMRT}

\section{Introduction}

Over the past two decades, radiation delivery has evolved from conventional external beam two-dimensional RT (2DRT) to three dimensional conformal RT (3DCRT). In a further advance, intensity-modulated RT (IMRT) uses computed tomography-based planning and delivery of radiation, aided by computerized optimization of the intensities of multiple beams. This improves the ability to tightly conform the treatment volume to concave tumor shapes, while minimizing doses to organs at risk (OAR), it can provide significantly better tumor target coverage and sparing of sensitive normal tissue compared with 3DCRT [1], [2].

Conformal external radiotherapy is based on the extensive use of modern medical imaging techniques, efficient dosimetric software, accurate patient positioning methods, stringent verification and quality control of procedures, aiming to increase tumor control by boosting tumor dose, reducing morbidity and sparing healthy tissues [3].

Three-dimensional conformal radiation therapy uses computed tomography planning to generate 3D volumes of a patients' anatomy. Using this information, multiply-shaped beams may be designed to deliver the dose to the intended target while relatively sparing critical structures. 3DCRT is limited to a geometric shaping of the beam so that its contour corresponds to the "beam's eye view” projection of the target. Usually the target is not geometrically well separated from surrounding normal structures (i.e., OARs). It often produces unacceptable plans for concave or irregular targets that are close to critical structures [4].

Intensity-modulated radiation therapy (IMRT) using dynamic or static multileaf collimators delivers highly conformal dose while sparing the surrounding normal structures. Using a conventional multileaf collimator can be made in a "dynamic" or "static" form.

In the dynamic form, the leaves at each gantry position are swept across the target while the beam is on and their speed determines the radiation fluency.

In static or segmental multileaf IMRT, each field consists of multiple segments with different intensities. These forms of IMRT are currently offered by most manufacturers of linear accelerators [5]-[7].

Intensity-modulated radiation therapy also has disadvantages resulting from the complexity planning and delivery processes, and the possibility of errors in planning or delivery that follow from that complexity. The heterogeneity of the delivered dose means that some areas of the tumor may receive a suboptimal radiation dose, and variations in patient positioning and anatomy may lead to perturbations in the delivered dose. Few comparative studies have been performed comparing the costs and resource use of IMRT against those of 2DRT and 3DCRT. Intensity-modulated radiation therapy required additional clinician input for delineating target volumes and additional physics time required to perform the more complex quality assurance necessary, although radiographer time was reduced compared with 2DRT. Assessment of the risks and benefits of IMRT is therefore important in determining its clinical utility [8].

The dose-volume-histogram (DVH) is a common tool to express the dose that is delivered to targets and organs-at risk (OAR). A DVH contains information about the doses delivered to partial volumes (either absolute or relative) of targets or OARs. However, DVHs do not provide spatial information such as the location of the high- and low-dose regions ("hot" and "cold" spots) inside the volume of interest (VOI) [9].

Patient-specific quality assurance (QA) is an essential step to guarantee accurate patient treatment in radiation therapy. Verification procedures are performed at various levels starting with the QA of machine-related parameters such as beam flatness and stability, accuracy of the leaf positions of a multi-leaf collimator (MLC), and accurate modelling of the linear accelerator at the commissioning stage of the treatment planning system (TPS). Verification procedures for 3D conformal radiation therapy (3DCRT) and intensity-modulated radiotherapy 
(IMRT) are commonly performed for an individual patient [10].

\section{Materials and Methods}

\subsection{Planning Systems and Radiotherapy Machine}

In our study, computerized Medical Systems (CMS) Inc.'s (St. Louis, MO) XiO software release 4.64 which is a three-dimensional treatment planning system that incorporates modern dose calculation algorithms for dose calculation was used to plan cancer patients. PRIMUS linear accelerator (3882) was used in this work. It is a multienergy machine (6 and 15 MV operating up to $200 \mathrm{MU} / \mathrm{min}$. The PRIMUS come standard with a 58-leaf, 3-D MLC comprising two parallel sets of 29 independent leaves each (29 leaf pairs) at isocenter, the outer most leaf pairs each measure $6.5 \mathrm{~cm}$ wide and the inner 27 leaf pairs each measure $1.0 \mathrm{~cm}$ wide, maximum overtravel 10 $\mathrm{cm}$ over the beam central axis for each leaf and minimum leaf gap $0.0 \mathrm{~cm}$ the leaf position is accurate only to within $2 \mathrm{~mm}$ was used for treatment delivery sessions.

\subsection{Acquisition and Simulation}

All patients underwent a CT scan with $2 \mathrm{~mm}$ slice thickness. After the patients scanning either prone or supine position the CT study has transferred to focal. Target volume definition had to be based on CT and magnetic resonance imaging (MRI) to create image fusion, the tumor volume such as gross target volume, clinical target volume and planning target volume (GTV, CTV and PTV) had been delineated as well as OAR included brainstem, optical nerves and lenses on the CT images, by the physician on each axial slice then CT slices were transferred to XiO 4.64 (Computerized Medical Systems, St. Louis, MO, USA) treatment planning system.

\subsection{Patient's Selection}

Twelve patients selected with different diagnosis brain tumor to be planned with IMRT and 3DCRT. The tolerance level for maximum dose was 6.0 Gy for lenses and 54.0 Gy for brain stem, and optical nerves [11]. The beam arrangement was determined by the size and location of the tumor. For 3DCRT plans used two or three wedge-paired fields, Non-co-planar fields were also used to optimize target dosing in some cases, five fields for IMRT planning were used. Prescription dose and normal-tissue constraints were identical for the 3DCRT and IMRT plans. Tumor and normal-tissue dose-volume histograms were generated for each plan. The IMRT and 3DCRT plans were then compared.

\subsection{Conformal Planning}

Treatment plans were created with $6 \mathrm{MV}$ photons. All fields were shaped at the beam's eye view to encompass the PTV shape using multileaf collimator (MLC). The treatment target volume included the PTV and an additional $0.7-\mathrm{cm}$ margin for beam penumbra in all directions. The treatment field's isocenter was positioned in the center of the PTV and the calculation point was taken at the treatment field's isocenter. Physical wedges (PW) and virtual wedges (VW) were used to modify the dose in the treatment plan and to perform dose homogeneity in PTV.

\subsection{Inverse-Planned IMRT}

Treatment plans were created for 6-MV photons with the same TPs with objective functions based on physical constraints. IMRT plans were generated using commercial inverse planning software. The beams are spread around the target with equispace and to avoid the opposing fields an odd numbers of the treatment fields were used.

\subsection{Treatment Planning Evaluation Tools}

The new TPs have many tools for qualitative and quantitative evaluation of the treatment plans.

The visual slice-by slice review of the treatment plans using isodose lines distribution can be used as a qualitative evaluation for the treatment plans. The qualitative evaluation is important to know the location of the hot and cold areas in the treatment plans. The quantitative evaluation included the maximum, minimum, mean doses 
and DVHs.

Dose Volume Histogram (DVH) was generated to evaluate the dose to the different structures in different treatment plans.

For PTV, the parameters, D98\%, D95\% and D2\% were used for plan evaluation, where D98\% and D2\% values are defined as the dose received by $98 \%$ and $2 \%$ of the PTV volume these two values are represented the maximum and minimum doses in the PTV, D95\% is target volume covered by $95 \%$ of the prescribed dose, for OARs, the mean and maximum dose for brain stem, optic nerve and lenses were used for treatment plan evaluation.

\subsection{Quality Assurance}

For the IMRT treatment planning where there were large numbers of treatment fields and each field divided into many segments. These special situations made it impossible to make manual calculation for the MUs as a quality assurance for the treatment plan calculation. Since a verification of dose distributions within a real patient is not possible, the phantom substitution method is often used [12]. Quality assurance for the calculated IMRT treatment plans were performed by transferring the plans to the phantom then point dose and dose distribution in the phantom were measured and compared to the treatment planning calculations. Water equivalent phantom, PTW RW3 and the $0.6 \mathrm{~cm}^{3}$ farmer-type ionization chamber were used for point dose measurements. For dose distribution the ionization chamber was replaced with the 2D-array [13] point dose and dose distribution measurements were performed at the machine isocenter with measurement depth of $15-\mathrm{cm}$. The phantom were scanned at the CT then transferred to the TPS, after an Conformal \& IMRT plans were performed and accepted for treatment by the physician and the physicist the verification process starting as following:

\subsection{Absolute Dose for All Treatment Fields}

The same phantom setup used with ion chamber at a plane perpendicular to the beam entrance direction for measuring absolute dose The measured point doses were compared to the average planned dose. An acceptance criterion for the absolute dose was developed; the absolute dose was considered adequate if the difference between the calculated and the measured dose was $<3 \%$. If the difference in point dose calculations lied between $3 \%$ and $5 \%$ it was accepted but if it was more than $5 \%$ it was rejected [14].

\subsection{Relative Dose for Each Treatment Field (the Field-Related Approach Method)}

Although the 2D-ARRAY is already calibrated in absorbed dose to water, normally each measurement must be corrected for different air and temperature. All treatment parameters were the same as for the real patient plan, except the gantry angle, which was normally set to $0^{\circ}$ for all beams. Dose distribution was calculated on a coronal plane at $15 \mathrm{~cm}$ depth. All fields are irradiated one after the other and measured by software Matrix Scan. Comparisons between calculated and measured isodoses were carried out by Verisoft [15] using widely used IMRT QA analysis tool, the gamma index, in which two dosimetric parameters, dose difference and distance-to-agreement (DTA), were frequently used to evaluate the agreement between planned and delivered IMRT fields. According to some researchers, a 3\% dose difference and $3 \mathrm{~mm}$ DTA in the planned and delivered IMRT fields constituted acceptable agreement between the two types of fields [16].

\section{Results}

As compared with the 3D-CRT plans, the IMRT plans provided superior target coverage, the minimum and maximum PTV doses With IMRT were $77.31 \pm 20.98$, and 107.9 \pm 2.94 respectively; for 3D-CRT were $75 \pm$ 16.99 , and $106.3 \pm 2.38$ respectively.

There was slightly a difference in max dose in PTV for IMRT and conformal as showed in Figures 1 and 2. Figures 3-5 show results of the coverage for PTV 98\%, PTV95\% and PTV2\% in IMRT were $94.6 \pm 2.3$, 96.56 \pm 1.67 and $106.5 \pm 1.87$ respectively; for 3D-CRT were $92.7 \pm 3.09$, $95.58 \pm 1.24$ and $104.45 \pm 2.73$ respectively.

Figures 6-9 showed maximum dose for brain stem, optic nerves and lenses. The IMRT plans also provided reduced doses to brainstem $(72.17 \pm 29.967)$ and lenses $(2.43 \pm 2.39)$ relative to the 3D-CRT plans for brainstem (73.05 \pm 34.59$)$ and lenses (7.65 \pm 23.24$)$. But optic nerves with IMRT significantly higher doses (38.86 \pm 32.15$)$ than 3D-CRT (27.57 \pm 31.45$)$. DVH for PTV and critical organs; brain stem, optic chiasm optic nerves and eyes 
PTV 98\%

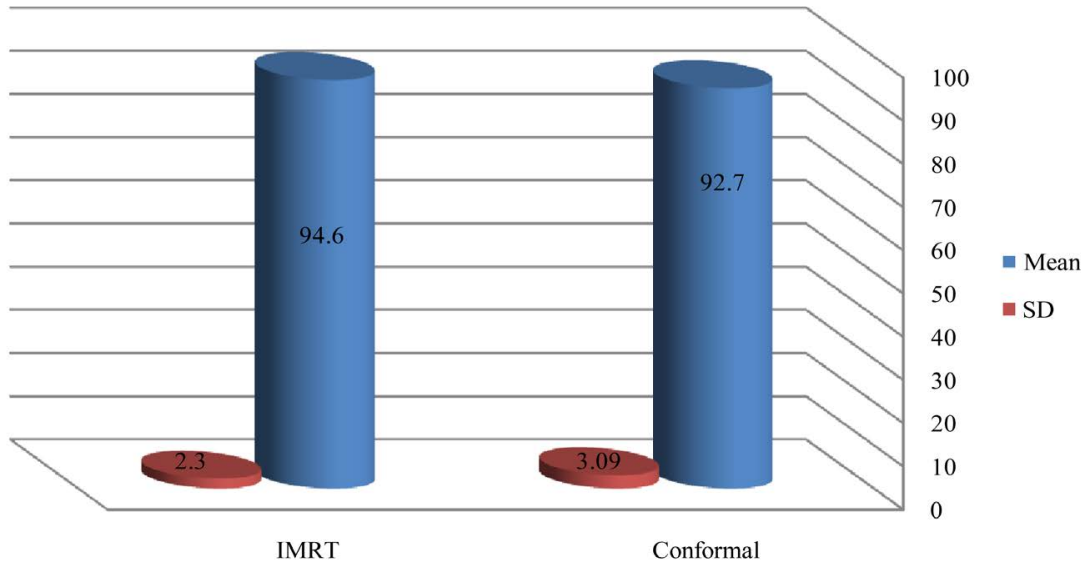

Figure 1. Mean and standard deviation (SD \pm ) PTV 98\% in IMRT versus 3D-CRT in twelve patients with brain tumor.

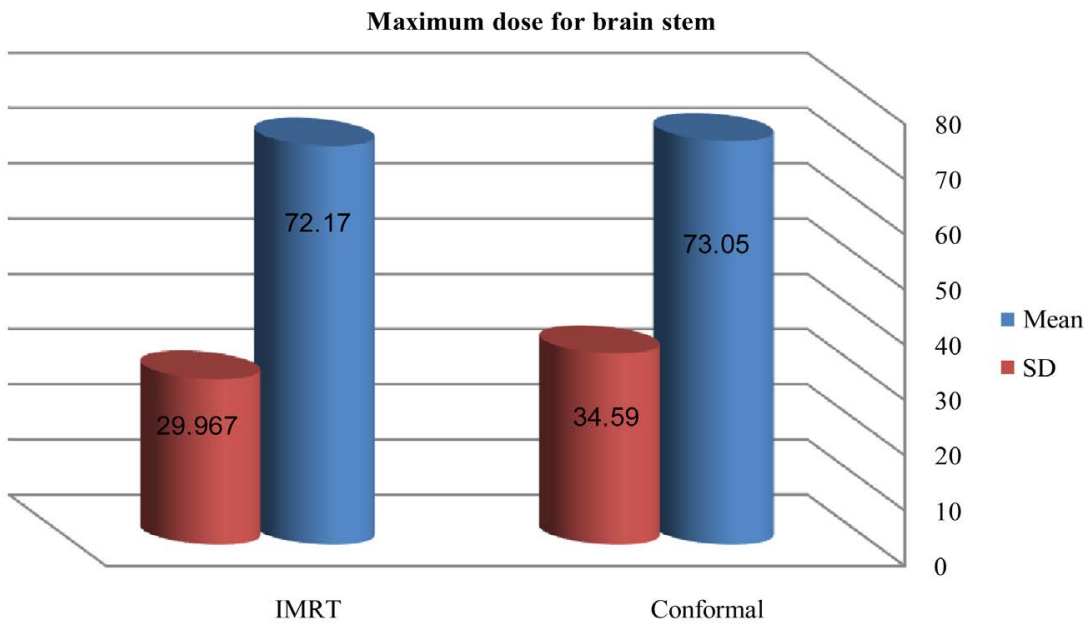

Figure 2. Mean and standard deviation (SD \pm ) max dose for brain stem in IMRT versus 3D-CRT in twelve patients with brain tumor.

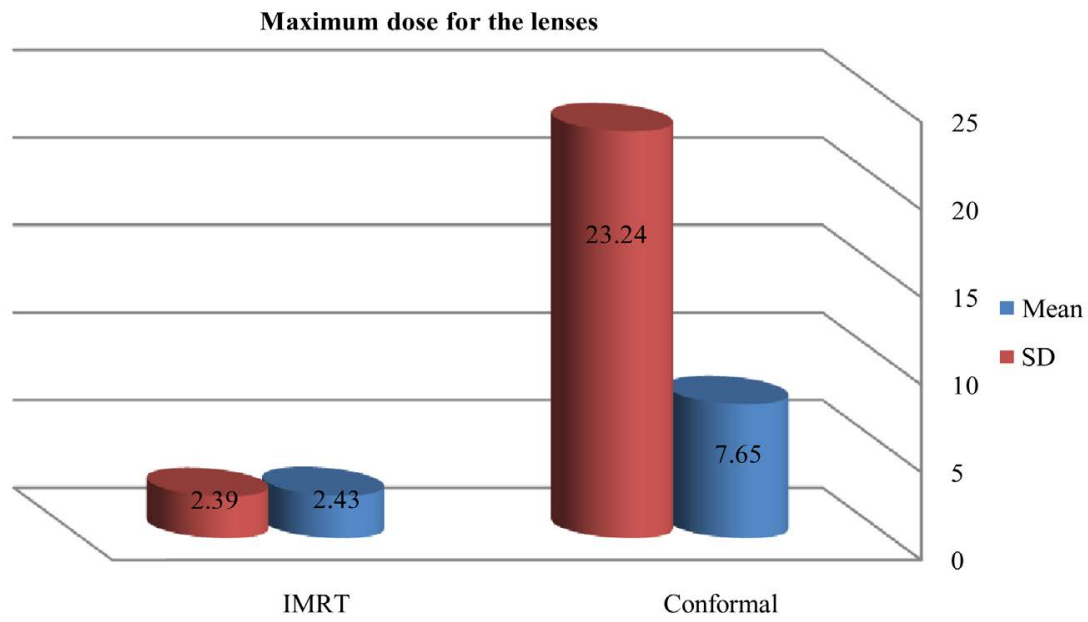

Figure 3. Mean and SD ( \pm ) max dose for lenses in IMRT versus 3D-CRT in twelve patients with brain tumor. 


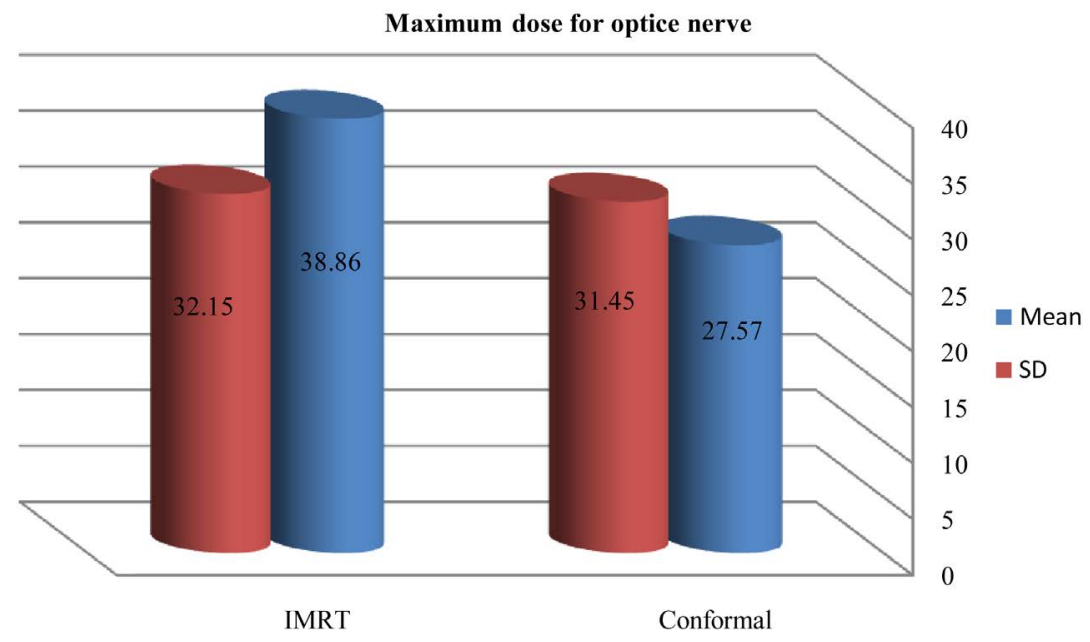

Figure 4. Mean and SD ( \pm ) max dose for optic nerve in IMRT versus 3D-CRT in twelve patients with brain tumor.

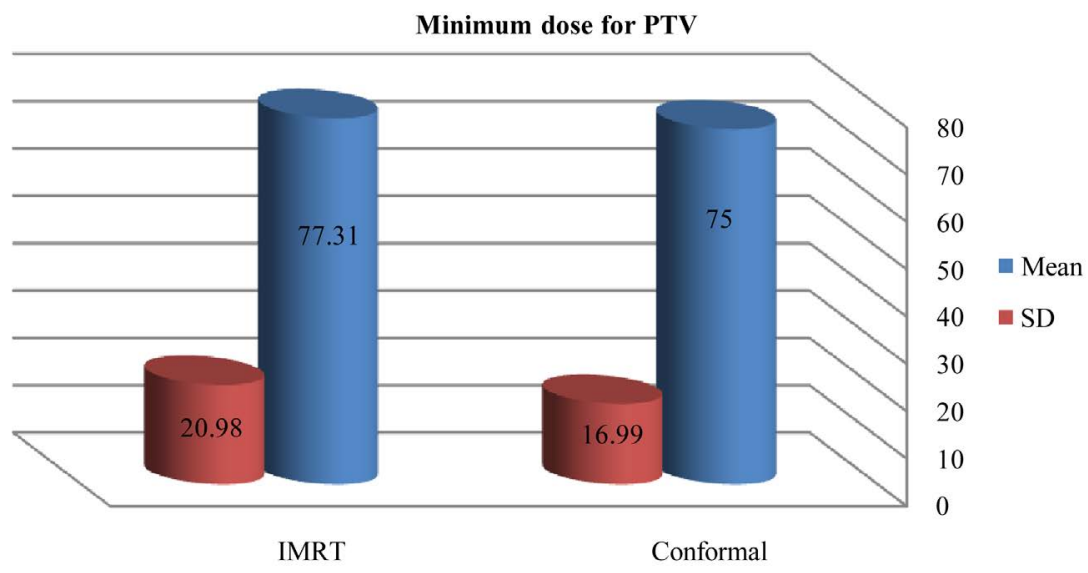

Figure 5. Mean and standard deviation ( $\mathrm{SD} \pm$ ) minimum dose for PTV in IMRT versus $3 \mathrm{D}-\mathrm{CRT}$ in twelve patients with brain tumor.

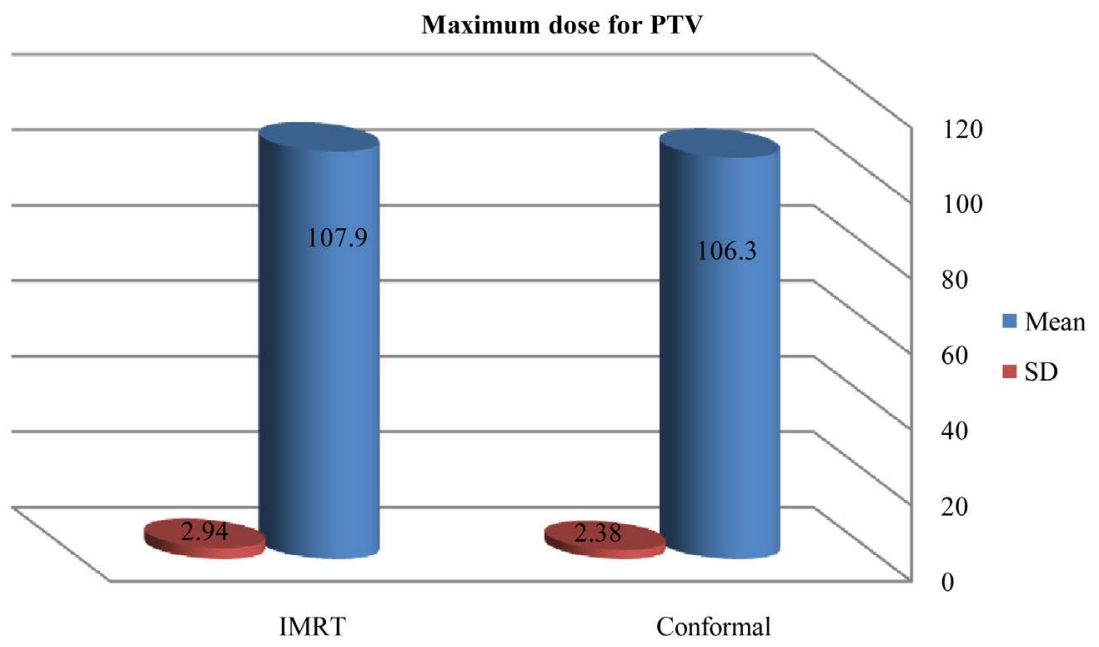

Figure 6. Mean and standard deviation ( $\mathrm{SD} \pm$ ) maximum dose for PTV in IMRT versus 3D-CRT in twelve patients with brain tumor. 


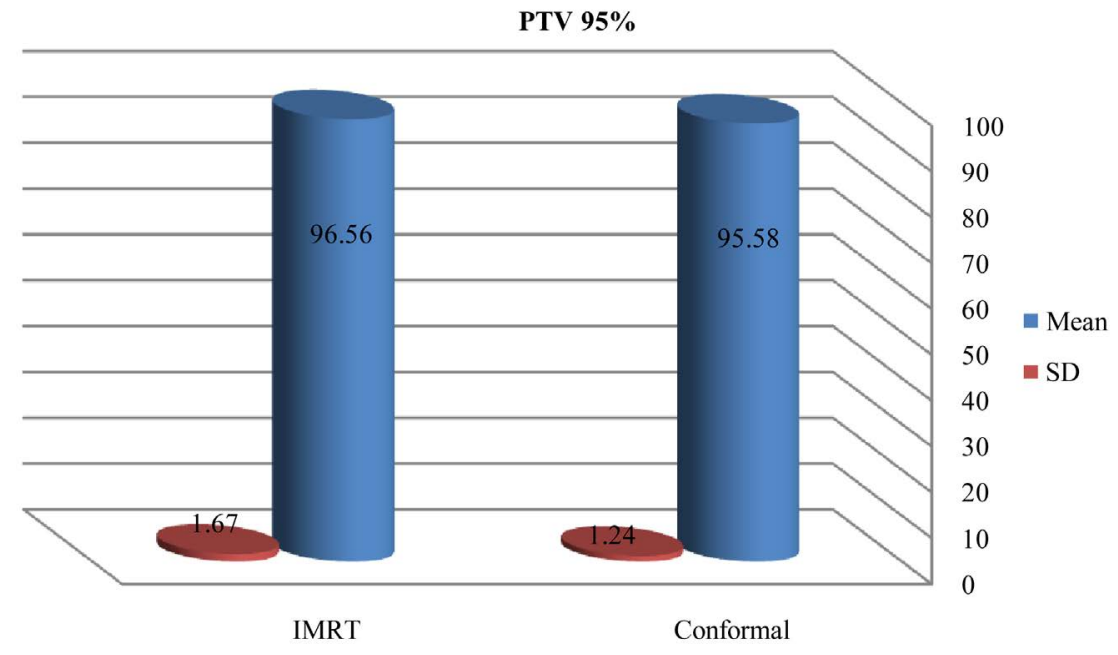

Figure 7. Mean and standard deviation (SD \pm ) PTV 95\% in IMRT versus 3D-CRT in twelve patients with brain tumor.

PTV 2\%

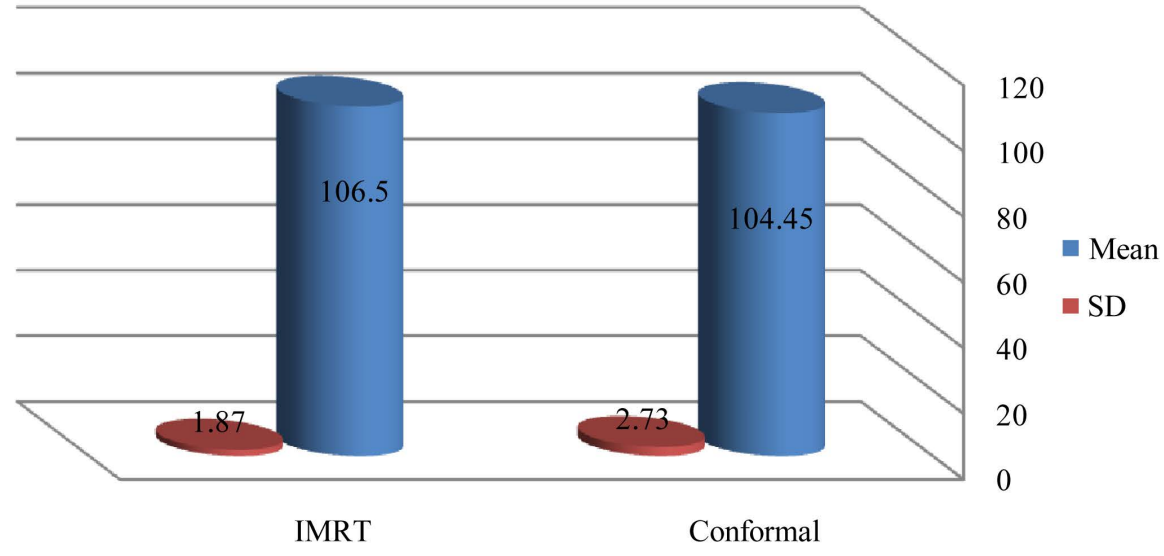

Figure 8. Mean and standard deviation (SD \pm ) PTV 2\% in IMRT versus 3D-CRT in twelve patients with brain tumor.

as shown in the Figures 10 and 11 isodose curves in a patient with brain tumor in three views between 3DCRT versus IMRT plan showed in Figure 11.

\section{Discussion}

We initially performed a dosimetric study to compare standard 3DCRT with IMRT. The results showing that the coverage of the PTV Figures 1-5 was better with IMRT than the 3DCRT, also there was significant decrease in the dose to OARs with IMRT Figures 6-8, especially for the organ distant in the tumor. The PTV coverage was acceptable if $95 \%$ of the volume were covered by $95 \%$ of the prescribed doses. This was comparable to PTV coverage achieved by the intensity-modulated treatment technique. 3DCRT plans were performed using two or three treatment fields with wedges in comparable with the IMRT plans which performed with five fields, this small number of the treatment fields given advantages to the 3D conformal technique where this will make small low-dose areas, less monitor units and short treatment time. This is an important because low-dose areas are suspected to induce secondary cancer as late toxicity and smaller number of monitor units implicates less scattered radiation [17]-[19]. In summary the differences between these two techniques are significant especially when PTV near OARs. If the PTV was far off OARs the 3DCRT technique will be adequate for a good and simple treatment plan. 


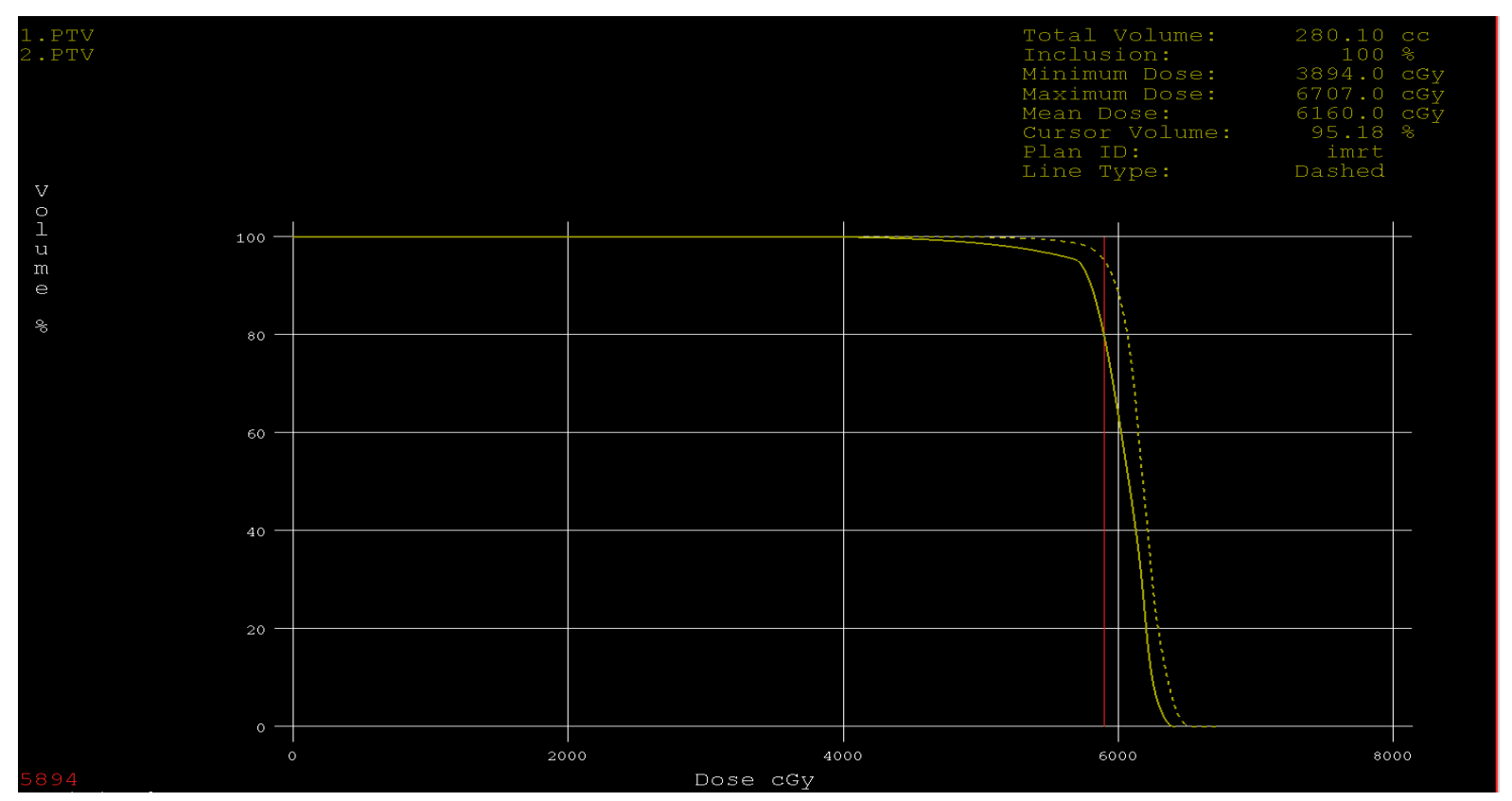

Figure 9. Dose volume histograms comparing: IMRT (dashed line) and 3DCRT (solid line) plans for PTV in a patient with glioblsatoma multiform (GBM).

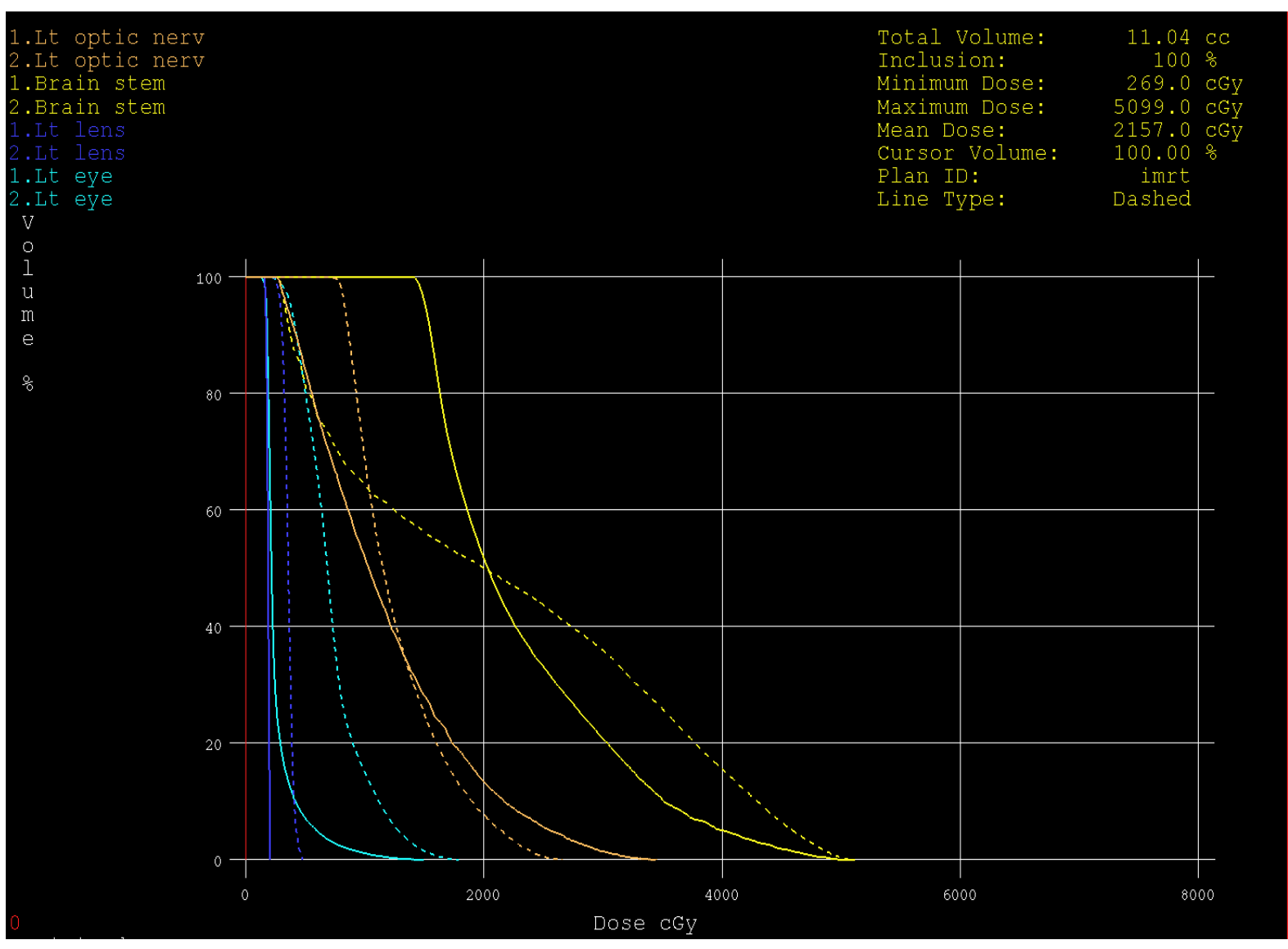

Figure 10. Dose volume histograms comparing: IMRT (dashed line) and 3DCRT (solid line) plans; for left optice nerve (brown), brain stem (yellow), left eye (light blue) and left lens (dark blue) in a patient with glioblsatoma multiform (GBM) in the left side of brain. 
3D-CRT

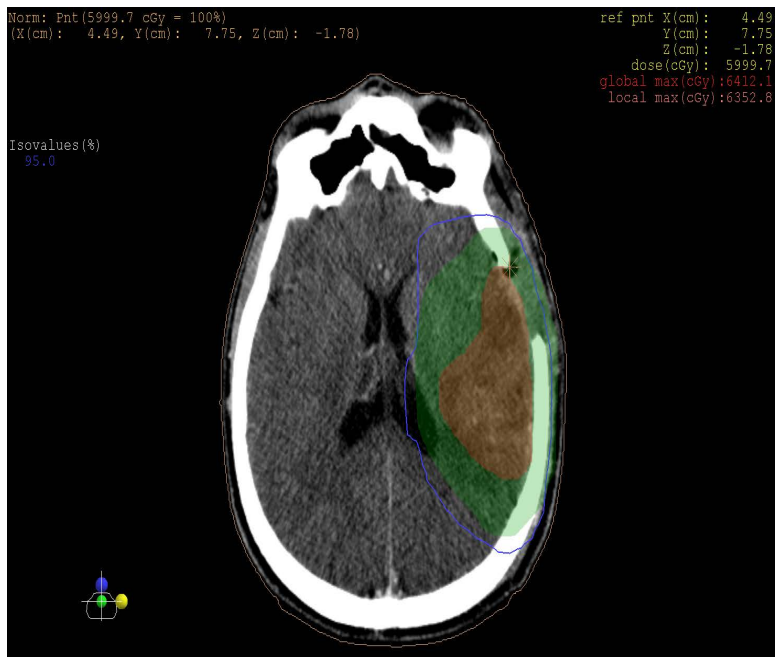

(a)
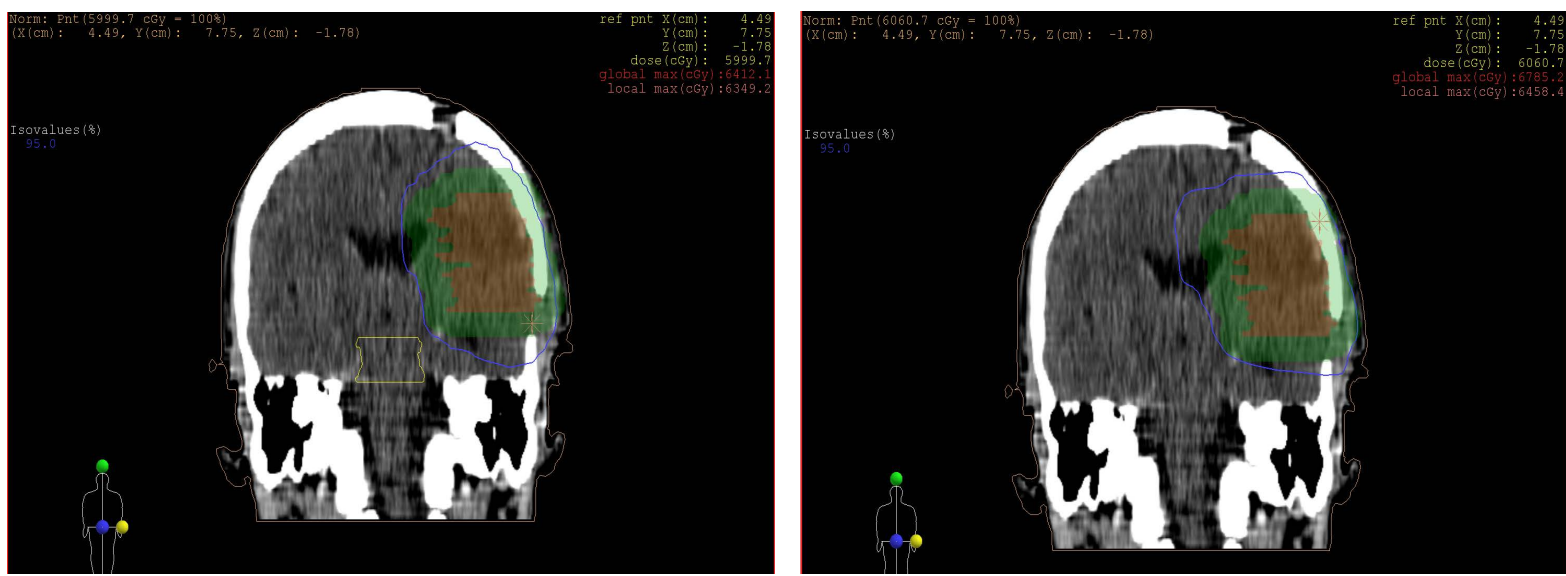

(b)
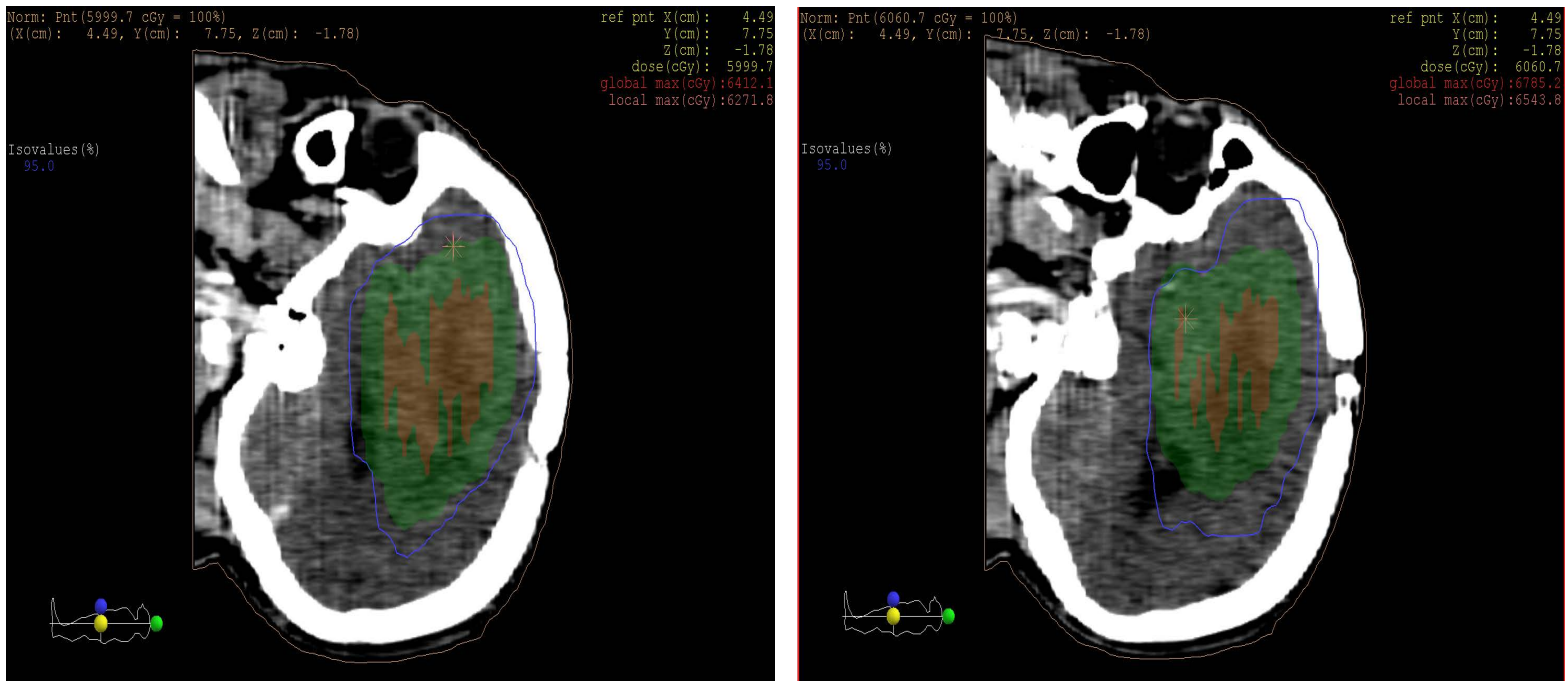

(c)

Figure 11. Isodose lines 95\% from prescribed dose of PTV (blue line) with (a) axial, (b) coronal and (c) sagittal views; left: 3DCRT and right: IMRT. PTV (Green wash) and tumor (Red wash). 


\section{Conclusion}

In the present study, target dose coverage with IMRT planning was better than 3DCRT planning. If PTV is distant to optical nerves, chiasm and brainstem 3D conformal technique can be applied, and if the PTV is nearby OAR intensity-modulated treatment technique should be used.

\section{References}

[1] Xia, P., Fu, K.K., Wong, G.W., Akazawa, C. and Verhey, L.J. (2000) Comparison of Treatment Plans Involving Intensity-Modulated Radiotherapy for Nasopharyngeal Carcinoma. International Journal of Radiation Oncology, Biology, Physics, 48, 29-37. http://dx.doi.org/10.1016/S0360-3016(00)00585-X

[2] Wu, Q., Manning, M., Schmidt-Ullrich, R. and Mohan, R. (2000) The Potential for Sparing of Parotids and Escalation of Biologically Effective Dose with Intensity-Modulated Radiation Treatments of Head and Neck Cancers: A Treatment Design Study. International Journal of Radiation Oncology, Biology, Physics, 46, 195-205. http://dx.doi.org/10.1016/S0360-3016(99)00304-1

[3] Tyng, C.J., Chojniak, R., Pinto, P.N.V., Borba1, M.A., Bitencourt, A.G.V., Fogaroli, R.C., Castro, D.G. and Novaes, P.E. (2009) Conformal Radiotherapy for Lung Cancer: Interobservers' Variability in the Definition of Gross Tumor Volume between Radiologists and Radiotherapists. Radiation Oncology, 4, 28. http://dx.doi.org/10.1186/1748-717X-4-28

[4] Goyal, S., Cohler, A., Camporeale J., Narra, V. and Yue, N.J. (2008) Intensity-Modulated Radiation Therapy for Orbital Lymphoma. Radiation Medicine, 26, 573-581. http://dx.doi.org/10.1007/s11604-008-0276-1

[5] Bhatnagar, A.K., Brandner, E., Sonnik, D., Wu, A., Kalnicki, S., Deutsch, M. and Heron, D.E. (2006) Intensity Modulated Radiation Therapy (IMRT) Reduces the Dose to the Contralateral Breast When Compared to Conventional Tangential Fields for Primary Breast Irradiation. Breast Cancer Research and Treatment, 96, 41-46. http://dx.doi.org/10.1007/s10549-005-9032-8

[6] Zelefsky, M.J., Fuks, Z., Hunt, M., Yamada, Y., Marion, C., Ling, C.C., Amols, H., Venkatraman, E.S. and Leibel, S.A. (2002) High-Dose Intensity Modulated Radiation Therapy for Prostate Cancer: Early Toxicity and Biochemical Outcome in 772 Patients. International Journal of Radiation Oncology, Biology, Physics, 53, 1111-1116. http://dx.doi.org/10.1016/S0360-3016(02)02857-2

[7] Heron, D.E., Gerszten, K., Selvaraj, R.N., King, G.C., Sonnik, D., Gallion, H., Comerci, J., Edwards, R.P., Wu, A., Andrade, R.S. and Kalnicki, S. (2003) Conventional 3D Conformal versus Intensity-Modulated Radiotherapy for the Adjuvant Treatment of Gynecologic Malignancies: A Comparative Dosimetric Study of Dose-Volume Histograms. Gynecologic Oncology, 91, 39-45. http://dx.doi.org/10.1016/S0090-8258(03)00461-X

[8] Tribius, S. and Bergelt, C. (2011) Intensity-Modulated Radiotherapy versus Conventional and 3D Conformal Radiotherapy in Patients with Head and Neck Cancer: Is There a Worthwhile Quality of Life Gain. Cancer Treatment Reviews, 37, 511-519. http://dx.doi.org/10.1016/j.ctrv.2011.01.004

[9] Guckenberger, M., Pohl, F., Baier, K., Meyer, J., Vordermark, D. and Flentje, M. (2006) Adverse Effect of a Distended Rectum in Intensity-Modulated Radiotherapy (IMRT) Treatment Planning of Prostate Cancer. Radiotherapy and Oncology, 79, 59-64. http://dx.doi.org/10.1016/j.radonc.2006.03.004

[10] van Elmpt, W., Nijsten, S., Mijnheer, B., Dekker, A. and Lambin, P. (2008) The Next Step in Patient-Specific QA: 3D Dose Verification of Conformal and Intensity-Modulated RT Based on EPID Dosimetry and Monte Carlo Dose Calculations. Radiotherapy and Oncology, 86, 86-92. http://dx.doi.org/10.1016/j.radonc.2007.11.007

[11] Boehmera, D., Bohsungb, J., Eichwurzelb, I., Moysb, A. and Budach, V. (2004) Clinical and Physical Quality Assurance for Intensity Modulated Radiotherapy of Prostate Cancer. Budach Radiotherapy and Oncology, 71, 319-325. http://dx.doi.org/10.1016/j.radonc.2004.02.016

[12] Wagner, D., Christiansen, H., Wolff, H. and Vorwerk, H. (2009) Radiotherapy of Malignant Gliomas: Comparison of Volumetric Single Arc Technique (RapidArc), Dynamic Intensity-Modulated Technique and 3D Conformal Technique. Radiotherapy and Oncology, 93, 593-596. http://dx.doi.org/10.1016/j.radonc.2009.10.002

[13] Xing, L., Curran, B., Hill, R., Holmes, T., Ma, L., Forster, K.M. and Boyer, A.L. (1999) Dosimetric Verification of a Commercial Inverse Treatment Planning System. Physics in Medicine and Biology, 44, 463-478. http://dx.doi.org/10.1088/0031-9155/44/2/013

[14] Leybovich, L.B., Sethi, A. and Dogan, N. (2003) Comparison of Ionization Chambers of Various Volumes for IMRT Absolute Dose Verification. Medical Physics, 30, 119-123. http://dx.doi.org/10.1118/1.1536161

[15] Venencia, C.D. and Besa, P. (2004) Commissioning and Quality Assurance for Intensity Modulated Radiotherapy with Dynamic Multileaf Collimator: Experience of the Pontificia Universidad Católica de Chile. Journal of Applied Clinical Medical Physics, 5. 
[16] Jursinic, P.A. and Nelms, B.E. (2003) 2-D Diode Array Analysis Software for Verification of Intensity Modulated Radiation Therapy Delivery. Medical Physics, 30, 870-879. http://dx.doi.org/10.1118/1.1567831

[17] Alvarez Moret, J., Koelbl, O., Bogner, L. and Quasi, I. (2009) MAT Technique and Secondary Cancer Risk in Prostate Cancer. Strahlentherapie und Onkologie, 53, 185.

[18] Fontenot, J.D., Lee, A.K. and Newhauser, W.D. (2009) Risk of Secondary Malignant Neoplasms from Proton Therapy and Intensity-Modulated X-Ray Therapy for Early-Stage Prostate Cancer. International Journal of Radiation Oncology, Biology, Physics, 74, 616-622. http://dx.doi.org/10.1016/j.ijrobp.2009.01.001

[19] Zwahlen, D.R., Ruben, J.D., Jones, P., Gagliardi, F., Millar, J.L. and Schneider, U. (2009) Effect of Intensity-Modulated Pelvic Radiotherapy on Second Cancer Risk in the Postoperative Treatment of Endometrial and Cervical Cancer. International Journal of Radiation Oncology, Biology, Physics, 74, 539-545.

http://dx.doi.org/10.1016/j.ijrobp.2009.01.051 


\section{Abbreviations}

2DRT: two-dimensional radiation therapy

3DCRT: three-dimensional conformal radiation therapy

CMS: computerized medical systems

CT: computed tomography

CTV: clinical target volume

DVH: dose-volume-histogram

DTA: distance to agreement

EPIDs: electronic portal imaging devices

GTV: gross target volume

Gy: gray

IMRT: intensity-modulated radiation therapy

MLC: multi-leaf collimator

MU: monitor unit

MRI: magnetic resonance imaging

$\mathrm{MV}$ : million volts

OAR: organ at risk

PTV: planning target volume

PW: physical wedges

QA: quality assurance

TPS: treatment planning system

VOI: volume of interest

VW: virtual wedges

D98\%, D95\% and D2\% were used for plan evaluation, where D98\% and D2\% values are defined as the dose received by $98 \%$ and $2 \%$ of the PTV volume these two values are represented the maximum and minimum doses in the PTV, D95\% is target volume covered by $95 \%$ of. 